\title{
El novel sujeto lector ante el texto académico: El difícil paso de la comprensión general a la especializada
}

\author{
When the Novice Reader Faces Academic Texts: The Difficult Path From General to \\ Specialized Comprehension
}

\section{O sujeito leitor novato diante do texto acadêmico: o difícil passo da compreensão geral para a especializada}

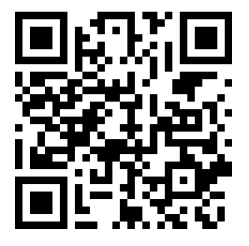

\author{
Lucila Lobato-Osorio \\ Universidad Icesi \\ Cali, Colombia \\ lucilalobato@gmail.com
}

https://orcid.org/0000-0002-3565-4886

Recibido • Received • Recebido: 07 / 07 / 2017

Corregido • Revised • Revisado: 05 / 11 / 2018

Aceptado • Accepted • Aprovado: 14/ 03 / 2019

\begin{abstract}
Resumen: El texto académico es un producto comunitario con un propósito científico, que utiliza estructuras y manifestaciones convencionales, y que desarrolla conceptos y vocabularios propios de las distintas disciplinas del conocimiento. Este trabajo se propone establecer que, por tales características, los textos académicos presentan dificultades para el sujeto lector inexperto, pues requieren de una comprensión especializada de lectura. Para conseguir el objetivo del artículo se presentan, primero, los resultados de un instrumento diagnóstico del nivel en la competencia de lectura con el que se está ingresando a universidad. Esta prueba sobre un texto de explicativoargumentativo está diseñada desde el enfoque del análisis del discurso y la lingüística textual, y se realizó durante el semestre 2017-1 a 733 estudiantes de nuevo ingreso de la Universidad Icesi de Cali, Colombia. Como segundo paso se revisan las características de los textos académicos que dificultan la comprensión de su contenido por quien recién se integra a este ámbito. Finalmente, se propone una serie de objetivos de aprendizaje que integrados a distintos cursos apoyarían al estudiantado para alcanzar la comprensión lectora especializada.
\end{abstract}

Palabras claves: Academia; comprensión lectora; educación superior; ingresantes a la universidad; textos universitarios. 
doi: http://dx.doi.org/10.15359/ree.23-2.14

URL: http://www.una.ac.cr/educare

CORREO: educare@una.cr

\begin{abstract}
The academic text is a conventionally structured and scientifically oriented community product that makes use of a specific set of concepts and vocabulary. This paper aims to establish that, due to such characteristics, academic texts present difficulties for the inexperienced reader as they require specialized reading comprehension. To achieve its goal, this paper presents the results of a diagnostic instrument of freshperson's reading competence level. The test was conducted on 733 new students from the Universidad Icesi in Cali, Colombia, during the first semester of 2017. Based on the discourse analysis and text-linguistics approach, the test was about an explanatory-argumentative text. On a second stage, the paper reviews the characteristics of the academic texts that difficult the reading comprehension by those who are newly integrated into the university. Finally, a series of learning objectives to be included in different courses is proposed in order to support the students' achievement of specialized reading comprehension.
\end{abstract}

Keywords: Academia; reading comprehension; freshmen; superior education; reading academic texts.

Resumo: O texto acadêmico é um produto comunitário com finalidade científica, que utiliza estruturas e manifestações convencionais, e desenvolve conceitos e vocabulários das diferentes disciplinas do conhecimento. Este trabalho tem como objetivo estabelecer que, por essas características, os textos acadêmicos apresentam dificuldades para o sujeito inexperiente de leitura, uma vez que requerem uma compreensão de leitura especializada. Para atingir o objetivo do artigo,

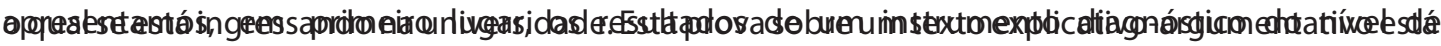

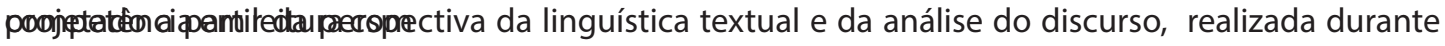
o semestre 2017-1 a 733 novos estudantes da Universidade ICESI Cali, Colômbia. Como segundo passo, são revisadas as características dos textos acadêmicos que dificultam a compreensão de seu conteúdo para aqueles que são novatos nesse campo. Finalmente, propõe-se uma série de objetivos de aprendizagem que, integrados em diferentes cursos, ajudariam aos estudantes alcançar uma melhor compreensão da leitura.

Palavras-chave: Academia; compreensão de leitura; educação superior; novatos universitários; textos universitários.

\title{
Introducción
}

La preocupación por la lectura en la universidad ha sido muy intensa en las últimas tres décadas. El inicio del aprendizaje en instituciones de educación superior saca a la luz problemas del estudiantado en la comprensión lectora que aparentemente deberían estar resueltos desde los niveles básicos de escolaridad. Sin embargo, es necesario considerar que el desarrollo de dicha competencia aún no termina y que, de hecho, la incorporación al ámbito universitario la hace más compleja y profunda. El objetivo de este trabajo es establecer que, debido a sus características, los textos académicos requieren de una comprensión de lectura especializada, la cual solo puede obtenerse a lo largo de la educación superior. Para conseguir este propósito, en primer lugar, se revisarán los resultados de una prueba diagnóstico de la competencia de lectura que refleja el tipo de comprensión lectora con el que se está ingresando a universidad. Después, se revisarán las características de los textos académicos y se analizarán las dificultades 
de comprensión que enfrenta quien recién se integra a este ámbito. Finalmente, se propondrá una serie de medidas, que incluye la persecución de objetivos de aprendizaje, con el propósito de ayudar a estudiantes para alcanzar la comprensión lectora especializada.

Desde finales de la década de 1980 empezaron a realizarse múltiples investigaciones al respecto de la comprensión lectora en el ámbito universitario. Iniciados en Estados Unidos, desarrollados en Inglaterra y recuperados en Iberoamérica, estos estudios pasaron de medir las habilidades de lectura y escritura en la educación superior a proponer estrategias de enseñanza de tales competencias comunicativas desde variadas tendencias teóricas y, finalmente a evaluar su aplicación (Carlino, 2003a, 2005; Carranza, Celaya, Herrera y Carezzano, 2004; Castelló, 2014; Riestre, 2006; Zanotto, 2016). El análisis, diagnóstico y puesta en marcha de programas remediales para estudiantes y de capacitación para docentes o personal formador de docentes ha generado una profusa bibliografía (solo en Colombia: González y Vega, 2012-2013; Pérez y Rincón, 2013; Salazar-Sierra et al., 2015). A pesar de todos estos esfuerzos, la situación concreta sobre la lectura en la universidad no parece mostrar un avance significativo; quienes ingresan a este nivel educativo siguen teniendo casi las mismas dificultades manifestadas desde hace, por lo menos, veinte años (Martínez, 1997). Como se verá a continuación, la comprensión lectora del nivel medio superior sirve para identificar algunas partes del texto, pero esto no es suficiente para abordar los textos académicos.

\section{Prueba de diagnóstico de comprensión lectora en Icesi}

El Proyecto de Competencias en Lectura y Escritura es una investigación llevada a cabo en la Universidad Icesi, de Cali, Colombia, por parte del Departamento de Lenguaje a cargo de Hoover Delgado Madroñero', que pretende identificar el nivel de lecto-escritura manejado tanto por estudiantes de nuevo ingreso como por quienes cursan los últimos semestres, de todas las facultades, a fin de reconocer las necesidades reales en estas áreas y establecer estrategias para mejorar su rendimiento. La investigación consiste en realizar una prueba diagnóstica sobre el nivel de competencia de lectura y de escritura de este estudiantado mediante la aplicación de un instrumento específico y el análisis cuantitativo y cualitativo de este mismo. La prueba se ha llevado a efecto, con algunos ajustes de criterios, durante cinco semestres. Particularmente, durante el semestre 2017-1 se aplicaron un total de 1045 pruebas de diagnóstico, de las cuales 733 fueron realizadas a la población total de estudiantes de nuevo ingreso y 312 a estudiantes del curso Programa de Desarrollo Profesional, que se imparte a estudiantes a punto de egresar. Para efectos de este trabajo solo se revisarán los resultados de la prueba de lectura de estudiantes de nuevo ingreso.

1 Se realiza en el marco del grupo de Investigación en Recursos y Teorías para el Aprendizaje (IRTA), Código COL0012201, de la Escuela de Ciencias de la Educación de la Universidad Icesi. 
doi: http://dx.doi.org/10.15359/ree.23-2.14

URL: http://www.una.ac.cr/educare

CORREO: educare@una.cr

Al respecto del instrumento de recolección de datos, la prueba de lectura se trata de un cuestionario de preguntas abiertas y de opción múltiple que debe ser contestado luego de la lectura de un texto de explicativo-argumentativo. Las preguntas están encaminadas a determinar la comprensión lectora desde el enfoque del análisis del discurso y la lingüística textual (Kintsch y Van Dijk, 1978; Van Dijk y Kintsch, 1983). Así, cada estudiante debe identificar la macroestructura o idea central del texto, las ideas principales que sostienen dicha idea; el propósito del texto, las intenciones comunicativas de la autoría, así como las voces ajenas que utiliza y, finalmente, la cohesión que logra dentro del texto a partir de la identificación de ciertos vínculos anafóricos y de los tonos empleados. La prueba está diseñada para ser respondida en una sesión de 90 minutos. La aplicación se realiza en la primera clase del curso de Comunicación Escrita (COE I) a fin de que brinde un diagnóstico exacto del nivel de la competencia al iniciar la formación universitaria. La evaluación de la prueba consiste en establecer el nivel de comprensión en que se encuentra cada estudiante a partir de la identificación correcta de las partes del discurso mencionadas. Así, la rúbrica establece quintiles que describen la situación de cada competencia.

En el caso de la competencia de lectura, el Nivel Cinco representa la comprensión total esperada; el estudiantado comprende con claridad el texto y es capaz de identificar y explicar todos sus elementos para relacionarlos con la intención comunicativa del autor o autora. EI Nivel Cuatro implica una comprensión lograda; en la que el estudiantado comprende el texto de manera clara y logra identificar casi todos los elementos, es capaz de explicarlos o justificarlos con exactitud a partir de la intención de su autoría. El Nivel Tres se caracteriza por una comprensión de lectura parcialmente lograda; en la cual, el estudiantado comprende de manera general el texto, pero únicamente logra identificar o explicar la mitad de los elementos de este. El nivel dos establece una comprensión no lograda, pues el estudiantado no identifica ninguno de los elementos del texto y no reconoce la intención comunicativa. Finalmente, el nivel uno se reconoce porque el estudiantado no responde a las preguntas y no hay elementos para determinar su comprensión del texto.

En un panorama general, en la aplicación de la prueba en el periodo 2017-1 casi la mitad (46\%) del estudiantado de nuevo ingreso, inscrito en cursos COE I, mostró un nivel de lectura cuatro. Otro amplio porcentaje de estudiantes de nuevo ingreso (38\%) tiene un nivel tres. Mientras que solo un $14 \%$ de estudiantes de nuevo ingreso tienen nivel cinco. Finalmente, un $2 \%$ tiene nivel dos. Estos resultados globales muestran que la mayoría de estudiantes de nuevo ingreso maneja una comprensión de lectura aceptable.

Sin embargo, en cuanto se analizan los componentes del análisis del discurso necesarios para la comprensión lectora, aparecen algunas dificultades específicas; particularmente, en aspectos que serán básicos en el ámbito universitario, según las características específicas que presentan los textos académicos: la inferencia de la idea central del texto, la estructuración adecuada de las ideas principales, así como la identificación del propósito del texto. 
Es decir, más de la mitad del estudiantado examinado tiene conflictos para inferir la tesis general del texto: $44.2 \%$ identifica la tesis central por medio de una redacción incorrecta; un $26.5 \%$ redacta una tesis, pero no es la correcta, pues no la relaciona con la intención comunicativa del texto; un $15 \%$ da cuenta de la tesis correcta aunque la redacción no es clara y un $13.3 \%$ infiere y redacta la tesis con claridad y relacionándola con la intención comunicativa; mientras que un $2 \%$ no responde la pregunta.

Por otra parte, el $10 \%$ deestudiantes de nuevo ingreso no identifica las tres ideas principales o no responde la pregunta; el $40 \%$ identifica solo una idea; el $31 \%$ identifica dos ideas; un $12 \%$ identifica las tres ideas, aunque las expresa con cierta dificultad y solo un 7\% identifica las tres ideas principales y las redacta con claridad.

Con respecto a la intención global del texto, un $2 \%$ no responde o no identifica ningún propósito; el $27 \%$ identifica un propósito incorrecto y tiene dificultades para redactarlo; el $26 \%$ redacta de manera adecuada un propósito, pero es incorrecto; el 11\% identifica el propósito comunicativo, pero lo expresa con dificultad y el $34 \%$ lo identifica y lo expresa claramente.

En cuanto a la identificación de las voces ajenas, la pregunta que se formuló versaba sobre la función que cumplía uno de los autores mencionados; se ofreció una respuesta de opción múltiple: el 7\% no respondió; el 34\% escogió la opción incorrecta y el 59\% seleccionó la opción correcta con respecto a la función de la voz ajena.

Todo esto implica que el estudiantado examinado comprende el contenido de un texto de manera superficial: son capaces de dar cuenta de los elementos concretos solicitados, aquellos que no requieren una inferencia o lectura crítica, sino que se pueden localizar en el texto; a saber: a) Las voces ajenas utilizadas en el texto; b) El tono empleado por este, es decir, la actitud que toma con respecto al tema abordado y c) Los vínculos anafóricos que darían cohesión al texto. Esto refleja que un alto porcentaje de estudiantes que están ingresando a la universidad no leen para comprender el texto de manera global, sino para responder preguntas específicas sobre él.

Los resultados de esta prueba de diagnóstico son interesantes para el objetivo de este trabajo, pues revelan con claridad las dificultades a las que se enfrentará el nuevo estudiantado, tomando como muestra al de la Universidad Icesi. El texto objeto de la prueba es de tipo explicativo-argumentativo, aunque no necesariamente académico o científico, pues se trató de no sesgar las respuestas a partir de las diferencias causadas por el conocimiento previo de una disciplina específica. Se escogió un texto que abarcase un tema conocido y familiar en el ámbito social y escolar, pero que entrañara la dificultad de un texto con un propósito más allá de la exposición de datos. Así, el autor trata de convencer al público lector de algo muy concreto: que un individuo no merece aspirar a un cargo público por haber cometido plagio. Como estrategia argumentativa, el autor ofrece definiciones, descripciones y clasificaciones de plagio. Esto confiere una complejidad muy específica al texto y, según los resultados de la prueba, muchos estudiantes parecen haberse detenido en este tipo de información, pero 
doi: http://dx.doi.org/10.15359/ree.23-2.14

URL: http://www.una.ac.cr/educare

CORREO: educare@una.cr

no la relacionaron con todo el contenido del texto y con la intención comunicativa de la que parte este; alcanzaron a comprender el tema (el plagio), pero no detectaron la tesis del autor, su construcción en las ideas principales, ni su intención última (quien comete plagio no debería tener un cargo público).

Quienes ingresan al nivel superior presentan un tipo de lectura que busca respuestas concretas y que no pretende vincular todos los componentes del texto. Esto puede deberse a que la educación básica prepara para lectura general y, hasta cierto punto, utilitaria. Sin embargo, leer de esta forma no es suficiente en el ámbito universitario. No se trata de que lleguen mal preparados, sino que necesitan aprender un nuevo tipo de lectura y escritura pertinente a una formación profesional; una comprensión requerida por los textos académicos utilizados en la universidad. Es decir, los nuevos grupos universitarios requieren formarse para una comprensión especializada.

Como se analizará a lo largo del próximo apartado, los textos académicos tienen unas características muy precisas: son comunitarios, convencionales de índole científica y con perspectiva disciplinar; las cuales exigen capacidades específicas a su público lector, más allá de identificar los contenidos temáticos del texto. Por esta razón es necesario ser conscientes de que la comprensión especializada conlleva un proceso que debe atenderse en el ámbito universitario.

\section{Características de los textos académicos}

Para que el estudiantado pueda adaptarse y superar con éxito la etapa de la educación superior requiere de un nivel especializado en la comprensión lectora a fin de entender, utilizar y elaborar unos textos particulares: los académicos. No se trata únicamente de saber leer con solvencia cualquier tipo de texto; en la universidad, el estudiantado se encontrará con unos textos escritos que serán su herramienta básica para adquirir conocimiento y formación profesional (Carlino, 2003b) y que se caracterizan por ser productos comunitarios, estar regidos por una serie de convenciones, tener un interés científico y pertenecer a una disciplina del conocimiento concreta (Teberosky, 2007).

Los textos universitarios se originan en el ámbito de una comunidad académica cuya tradición, sistema de organización, objetivos y prácticas giran en torno a la búsqueda, desarrollo y transmisión del conocimiento. Esta comunidad está formada por equipos investigadores, profesorado y estudiantes, quienes a través de sus productos construyen una red de relaciones que transmite constantemente ese saber. Los textos escritos son el vehículo principal que contiene, analiza y discute los conocimientos usados por cada miembro de la comunidad y, a su vez, los comunica ya mediante lecciones presenciales o remotas, ya por medio de otros textos escritos. Esto implica que tienen sujetos autores y públicos lectores definidos por su pertenencia a ese grupo; lo cual les otorga, necesariamente, unas relaciones y objetivos concretos. 
Precisamente por su procedencia y alcance académicos, sostenemos aquí que los textos utilizados en la educación superior son elaboraciones convencionales. Es decir, todo texto producido en ese ámbito se apega a ciertos parámetros de contenido, estructura, relaciones y propósitos acordados entre la misma comunidad a fin de que sea posible comprender, utilizar y replicar cada texto por todos los miembros del grupo. Las convenciones académicas pueden aplicarse al texto en su función, su tipología y su manera de expresar los contenidos. Siguiendo a Vidal-Moscoso y Manriquez-López, las funciones que cumplen estos textos son múltiples y se presentan de forma exclusiva o combinada; pueden ser instruccionales, informativos y formativos. Los textos son instruccionales cuando enseñan la forma en que se realizan ciertas prácticas, metodologías, o sistemas. Son informativos allí donde dan a conocer conceptos, ideas teorías, fórmulas o principios científicos. Asimismo, son formativos cuando sirven para fundamentar, aplicar o razonar sobre algún tema o teoría propios de la disciplina a la cual pertenecen (Vidal-Moscoso y Manriquez-López, 2016). Por su parte, en tanto discursos con funciones convencionales, los textos académicos pueden presentarse por medio de diversos géneros: manuales, libros, capítulos de libros, artículos de investigación, informes, tesis y otros. Esto permite al público lector de la comunidad reconocer la manera en que será abordado su contenido, saber cómo está organizado e intuir sus propósitos incluso antes de leerlo. La convencionalidad de este tipo también define la forma en que el autor o autora transmite la información en cuanto a vocabulario, estructuras sintácticas, elementos de cohesión, tono e intención, entre otras maneras de expresión.

Por otro lado, como parte de los parámetros establecidos por la comunidad universitaria, los textos académicos tienen un interés científico. Según Fuster (2016), esta característica les otorga su diferencia específica con respecto a otros textos de otros ámbitos, que también podrían ser comunitarios y convencionales. Los textos académicos están elaborados con el propósito de expandir el conocimiento a partir de criterios sistemáticos y regulados basados en los métodos, enfoques y procedimientos científicos. Al tiempo que presentan los resultados de un proceso de indagación científica, los textos académicos plantean conceptos, tesis, teorías y debates que involucran a otros textos de diferente espacio y tiempo, e incluso proponen escenarios hipotéticos o probables. Es decir, presentan información que debe comprobarse o, por lo menos, pretende convencer al público lector de la comunidad sobre su posibilidad o pertinencia. En los textos académicos se genera la discusión, revisión, problematización y diálogo entre distintos puntos de vista, incluso sin pretender tener la solución al problema estudiado. En ese sentido, tales escritos tienen contenidos y estructuras que muestran explícitamente su proceso de indagación a partir de recursos como la exposición y la argumentación; en ellos se prefiere mostrar al objeto de estudio o problema como el centro de la investigación por encima de las opiniones de quien lo escribió, aunque esta sea quien proponga hipótesis, tesis, ideas y objetivos -explícitos o implícitos- que guían tanto el proceso como la comunicación escrita de este y de sus resultados. 
doi: http://dx.doi.org/10.15359/ree.23-2.14

URL: http://www.una.ac.cr/educare

CORREO: educare@una.cr

Como última característica de los textos académicos, es necesario considerar que el abordaje del conocimiento se ha realizado, por tradición, a partir de disciplinas claramente delimitadas, que expresan de manera diferenciada su forma de observar y comprender los fenómenos de la realidad a partir de sus intereses específicos. Cada disciplina del saber se trata de una concepción del mundo determinada por estructuras específicas que se convierten en "un método especial de construir el conocimiento" (Henao-Salazar, Londoño-Vásquez y FríasCano, 2014, p. 34). En la universidad contemporánea las disciplinas se desarrollan y relacionan en diversos grados de interdependencia. Así, los textos académicos tienen rasgos particulares determinados por las disciplinas que los generan y emplean. Esta característica se refleja en el vocabulario, los conceptos, las fórmulas y los ideales teóricos, técnicos y prácticos construidos por cada materia del saber. Es en ese el marco disciplinar que los textos cobran un mayor significado para el desarrollo de los conocimientos y habilidades buscados por la educación superior, pues esto los ancla en temáticas, fórmulas, tradiciones y formaciones distintivas y específicas. Por lo anterior, comprender a cabalidad los textos académicos disciplinares requiere necesariamente pertenecer a esa comunidad universitaria concreta y dominar los elementos lingüísticos, discursivos, estructurales, intertextuales y contextuales que los constituyen (Marín, 2007).

Todas estas características de los textos académicos resultan relevantes porque los diferencian de todos los otros textos con los que el sujeto ingresante a la universidad haya tenido anterior contacto. También significan un desafío doble para su competencia lectora: exigen una comprensión general bien desarrollada (reconocer tanto la información como su relación con la intención comunicativa) y, al mismo tiempo, requieren una comprensión especializada que le permita captar los detalles convencionales, científicos y disciplinares de su contenido.

\section{Dificultades de estudiantes ante los textos académicos}

Quienes se incorporan a la vida universitaria han pasado por un largo proceso de aprendizaje gramatical y pragmático sobre el lenguaje, pero aún no dominan algunos aspectos básicos para la comprensión lectora satisfactoria, como se ha hecho evidente a partir de los resultados de la prueba diagnóstica de la Universidad Icesi. Esto implica que no cuentan con las habilidades de comprensión lectora requeridas por los textos del nivel superior (Carlino, 2003b; Estienne y Carlino, 2004; Vidal-Moscoso y Manriquez-López, 2016); lo cual "significa un verdadero obstáculo para el aprendizaje del contenido de cualquier ciencia, por mínima que sea su complejidad" (Ugarriza, 2006, p. 32). Por si fuera poco, estos lectores y lectoras tampoco utilizan las estrategias clave para incorporarse a una comunidad basada en textos con características tan complejas como las revisadas anteriormente. Esto podría pasar inadvertido para alguna parte del profesorado, al punto de soslayar que los textos académicos requieren mayor presentación o explicación. Pero, desde la perspectiva estudiantil, representan un verdadero reto. Partiendo de las características de tales textos, en los siguientes apartados se detallarán las dificultades a las que se enfrentan los noveles sujetos lectores en la educación superior. 


\section{A. Confusión de los tipos de géneros discursivos académicos}

Los ingresantes a la universidad desconocen las diferentes formas discursivas en que se pueden manifestar los textos académicos; esto complica su comprensión en la medida en que no saben qué esperar de cada texto ni de cada género que leen. Tampoco pueden reconocer relaciones entre otros textos o entre quien lo escribió y el tema; o entre el texto y ellos o ellas como sus sujetos receptores. Al obviar tanto la estructura como las relaciones, no buscan reconstruir el significado del texto, ni determinar información más relevante o inferir los supuestos que están implícitos (Mateos, 2009). Todo lo cual provoca una exclusión de su interés por el contenido y propósitos del texto, porque no alcanzan a ver la función que este desempeña en su propia formación profesional, social o personal (Van Dijk, 2010).

Por otro lado, este desconocimiento de los diversos tipos de textos a los que pueden enfrentarse impide al estudiantado distinguir los tipos de discursos más comunes en los géneros académicos: la exposición y la argumentación, que conllevan sus propias complicaciones como la abstracción, la crítica y la valoración, con los cuales no han tenido contacto hasta ahora (Mateos, 2009; Teberosky, 2007). Sin una guía, aportada por el género del texto, el lector o lectora carece de elementos para comprender un texto académico con la profundidad requerida en este momento de su formación.

A esto hay que añadir la fragmentación de los textos causada por el uso de fotocopias, documentos digitales o ligas electrónicas que el personal docente les entrega y que eliminan la idea de la materialidad o integralidad de los textos que leen (Carlino 2003b). Los textos fragmentados priorizan la presentación de contenido concreto para las clases y, generalmente, se presentan sin datos bibliográficos, sin elementos paratextuales y sin explicación de su género u origen; todo lo cual impide que quienes ingresan a la universidad construyan una visión global del texto, así como su concepción disciplinar y contextual (lugar de producción, autor o autora, propósito del texto, público destinatario, etc.). El estudiantado empieza a leer solo partes de libros, de artículos, de monografías; eso los conduce a ignorar elementos básicos para conocer el universo conceptual de cada materia o asignatura.

\section{B. Incapacidad para buscar y reconocer los objetivos autorales}

Por lo general -y debido a los hábitos aprendidos en la educación previa, según se vio en la prueba diagnóstica presentada- quienes ingresan a la universidad leen los textos académicos buscando información o respuestas específicas solicitadas por el profesorado; sin embargo, incluso esto les resulta complicado, porque entre los datos concretos pueden encontrar explicaciones, historias, ejemplos, polémicas y diversas secuencias discursivas que el autor o autora considera pertinentes para su objetivo en el texto. 
doi: http://dx.doi.org/10.15359/ree.23-2.14

URL: http://www.una.ac.cr/educare

CORREO: educare@una.cr

Por ello es necesario que tomen en cuenta que quien escribió tuvo un objetivo concreto para la construcción de ese texto y que determinó el contenido, la expresión y las relaciones. Además, a decir de Riestre (2006), las personas lectoras noveles no se incluyen en ese objetivo planeado por la escritora o el escritor, pues no se asumen como sujetos receptores del texto, aunque lo son por formar una parte de la comunidad universitaria. Al ignorar la importancia de la intención del texto, pierden tiempo valioso, y ven frustrados sus intentos de aprendizaje y compresión del texto porque están leyendo indiscriminadamente.

\section{Impericia para establecer la idea central o tesis del texto}

Otra de las dificultades estudiantiles para leer los textos académicos, también derivada de su formación básica, es no poder inferir con claridad la idea central del texto. Esta identificación solo se logra en un nivel avanzado de la competencia lectora porque requiere de varias operaciones: seleccionar y sintetizar el contenido del texto; valorar la información obtenida y crear una representación ya sea mental o escrita de la idea o tesis que da sentido al texto (macroestructura en términos del análisis del discurso: Kintsch y Van Dijk, 1978; Van Dijk y Kintsch, 1983; Van Dijk, 1983). Para el ente lector es complicado porque no basta comprender el texto, además ha de poner en juego su experiencia previa, sus conocimientos sobre el tema y sus propios objetivos de lectura.

De hecho, esto podría no ser suficiente para la lectura de los textos académicos de interés científico, en los que el estudiantado debe distinguir los contenidos, interpretaciones, supuestos y debates que están unidos por un hilo conductor: la tesis o idea principal del texto. Estas construcciones discursivas involucran las ideas o propuestas, ante las cuales el sujeto lector mismo debería fijar una postura utilizando su conocimiento. En este sentido, las investigaciones llaman la atención de la necesidad de que el sujeto lector reconozca la "dimensión polémica" de tales textos (Velásquez, Cornejoy Roco, 2008), pues quienes escriben recurren a la argumentación para persuadir de sus propuestas. Un sujeto lector ingenuo o desinteresado que ignora la idea central del texto no solo está perdiendo una comprensión completa de este, sino también se aleja del sentido comunitario de la lectura académica, porque deja de participar en el ciclo de la construcción del conocimiento.

\section{Ineficacia para distinguir y analizar las ideas principales planteadas por el texto}

Una dificultad aún más compleja es la selección y jerarquización de aquellas ideas que conforman la construcción discursiva del texto académico surgidas desde una tesis o idea central. Estas acciones son un reto para quien recién ingresa a la universidad porque debe partir, necesariamente, de una comprensión general del texto y de la identificación de la intención y la propuesta central de su autor o autora; es decir, forman parte de un proceso de comprensión 
al que quizá no está habituado o no ha dominado aún, según se presentó en la prueba diagnóstica. Por si esto fuera poco, debe desentrañar esas ideas principales establecidas entre definiciones, narraciones, exposiciones y argumentaciones, o reelaborarlas cuando no son del todo transparentes o accesibles, pues están expresadas en una "retórica académica" utilizada por este tipo de escritura. Esta práctica es resumida por Marín (2007, p. 65) en las siguientes formas de expresión: "los recursos de argumentación oculta, la mitigación de las aserciones, la reticencia para afirmar abiertamente, las fórmulas de cortesía científica, la evidencialidad, las causalidades poco explícitas, las variadas formas de la incerteza". Tales maneras de expresión tornan ininteligibles los textos universitarios para quien, a decir de la propia Marín (2007), es doblemente inexperto o inexperta: tanto en la disciplina como en el uso retórico de los textos. Sin duda, este es uno de los conflictos más comunes entre estudiantes en cuanto a comprensión lectora, al complicado proceso de reflexión crítica que requiere la reconstrucción mental de tales ideas, se añade la barrera lingüística y discursiva.

\section{E. Dificultad para determinar las relaciones de intertextualidad de los textos}

Como ya se estableció, la intertextualidad es uno de los elementos definitorios de la escritura académica porque en ella se condensan todas sus características esenciales: comunitaria, convencional, de interés científico y disciplinar. Un texto académico implica un diálogo que quien lo escribió establece con otros sujetos autores a partir de los textos citados; sin embargo, para la persona ingresante a esa comunidad, dicho diálogo puede resultar ajeno e incomprensible, lo que provoca desinterés por participar en él (Riestre, 2006).

Además, distinguir la voz autora frente a otras voces, identificar las diversas posturas y determinar las funciones de todas ellas en el texto es un desafío mayúsculo, pues significa el final de un proceso de comprensión que requiere atención y profundización en el discernimiento de las ideas expresadas en el texto. Para Mateos (2009), la persona lectora debe realizar la síntesis de múltiples voces, comprender las diversas funciones de esas voces, al tiempo que construye un significado propio o una voz propia que las integre a todas. Adicionalmente, los géneros escritos de cada disciplina tienen sus propios sistemas o formatos de citación para incluir las referencias de los textos mencionados, lo cual representa la necesidad de descifrar un código acordado y establecido por otras personas, frecuentemente desconocido por quien lee de manera novata.

Ante todas estas complejidades, la solución más utilizada por los sujetos lectores ingresantes a la universidad es ignorar tanto las relaciones de intertextualidad como los sistemas de citación (Carlino, 2003b); lo cual se convierte en un problema serio porque dejan de comprender el texto en su totalidad, dedicándose únicamente a recabar información o datos que les parecen útiles sin detenerse a afrontar los verdaderos aportes del texto académico para su integración a su disciplina y para su formación profesional. 
doi: http://dx.doi.org/10.15359/ree.23-2.14

URL: http://www.una.ac.cr/educare

CORREO: educare@una.cr

\section{F. Desconocimiento del vocabulario especializado y disciplinar}

Un inconveniente mayúsculo que enfrenta quien emprende la lectura de un texto académico es el desconocimiento de una serie de palabras que le impiden avanzar incluso en el primer nivel de comprensión, el literal (Giraldo, 2011); al desconocer los términos técnicos o incluso observar que una palabra conocida es utilizada de una forma particular, el proceso de lectura se vuelve tortuoso. Si a esto se añade la falta de hábito de consultar diccionarios o glosarios especializados, la comprensión del texto es difícil e ineficaz. Desgraciadamente, esta dificultad suele ser inadvertida por el profesorado y equipos de investigación, pues aparenta pertenecer a una comprensión básica que se supone cubierta en la educación anterior. Sin embargo, cobra importancia, si consideramos que el estudiantado está incorporándose apenas a un sistema lingüístico (el argot científico y disciplinar), cuyos referentes están establecidos y convenidos de antemano y que, por tanto, requieren una guía incluso en lo más elemental, como es el uso concreto del lenguaje en la respectiva disciplina.

Es decir, una dificultad como la falta de vocabulario tiene su origen en la formación estudiantil solo de manera parcial, pues incluso palabras conocidas, involucradas en la formación de un concepto científico ( $y$, por ende, dotado tradición, complejidad y un uso específico en una disciplina particular) pueden adquirir un significado inusual. Por ejemplo, puede conocer la definición de "esfera"; pero, este término variará de significado según esté ubicado en un contexto disciplinar como el de la física, el de la sociología o de la mercadotecnia. En ese momento, el estudiantado puede perder con facilidad el ritmo de la lectura y no comprender ni siquiera lo más elemental: las palabras.

\section{G. Inexperiencia en las prácticas y sistemas de la disciplina de su carrera o programa de estudios}

Una dificultad más compleja es la que involucra comprender los conceptos, metodologías y sistemas de organización de la disciplina donde el estudiantado empieza a desarrollar sus estudios. Además de encontrarse con nuevos términos lingüísticos y particulares formas de expresión, debe incorporarse a una disciplina específica que hasta entonces desconocía casi en su totalidad. Esto implica que no basta que tenga una buena comprensión lectora, requiere de un proceso de adaptación a su disciplina concreta y a sus modos de trabajo.

Al mismo tiempo, el interés científico desde una disciplina específica requiere de un "pensamiento conceptual" complejo (Marín, 2007), que el estudiantado novel aún no desarrolla porque carecen de los elementos para establecer relaciones de paralelismo, inclusión, simultaneidad, simetría y causalidad entre definiciones y conceptos.

Tampoco cuenta con un dominio de ese campo de conocimiento; no comprende sus explicaciones ni puede proporcionar otras porque aún no forma parte de esa comunidad que 
propone, articula, analiza e incrementa los conceptos de su área. Así, el estudiantado podría desarrollar capacidades de lectura especializada a la par que amplía su conocimiento en su área disciplinar a lo largo de la educación superior, pero no desde el inicio de esta (Carranza, et al., 2004). Sin embargo, "no todos los docentes -ni los propios estudiantes- son conscientes de la necesidad de iniciar, formalmente, la enseñanza-aprendizaje del discurso propio de cada disciplina" (Cassany y Morales, 2009, p. 110).

\section{Conclusiones}

Luego de todo lo revisado, podría asegurarse que las dificultades de lectura de los textos académicos de estudiantes que ingresan a la universidad se deben al bajo rendimiento en la competencia lectora, desde sus dos grados: La comprensión general y la compresión especializada. La primera abarca la enseñanza de esta competencia a un nivel avanzado, que se completa cuando el sujeto lector es capaz de inferir la idea principal, organizar las ideas principales, reconocer los objetivos de la autoría e identificar las voces ajenas. Como se vio en la prueba diagnóstica presentada, aunque no todo el estudiantado alcanza este nivel, la formación está diseñada para llegar hasta ese punto. Sin embargo, las dificultades en esta comprensión general no están relacionadas únicamente con deficiencias en ella misma; además es necesario puntualizar que tal competencia aún está en desarrollo, sobre todo si se considera la lectura como un proceso dinámico e interactivo (Carlino, 2003a, 2005).

Es factible asegurar que la parte más compleja de dicho proceso inicia en la educación superior, en especial, si se toman en cuenta las características y exigencias de los textos académicos que hemos revisado a lo largo de este trabajo. Con respecto a las dificultades relacionadas con la comprensión especializada, surgen por la desadaptación al ámbito académico en el que encuentra el estudiantado; se explican desde la concepción de la lectura como un proceso sociocultural que requiere de un ambiente específico para llevarse a cabo de manera integral (Van Dijk, 2010). En este trabajo se presentaron las dificultades de comprensión que padece el estudiantado por no pertenecer todavía a la comunidad disciplinar específica y obstaculizan la comprensión especializada: la confusión de los tipos de géneros discursivos académicos, el desconocimiento del vocabulario especializado y disciplinar, la inexperiencia en las prácticas y sistemas de la disciplina de su carrera o programa de estudios, y la dificultad para determinar las relaciones de intertextualidad de los textos.

Entonces, para mitigar estas dificultades en la lectura de textos académicos se requiere continuar con una formación particular en la competencia de comprensión, así como permitir una adaptación más sólida al ámbito universitario, particularmente desde la disciplina específica del nuevo grupo estudiantil. A este respecto, se han propuesto diversas estrategias para llevarla a cabo. La "alfabetización académica", configurada en América Latina por Carlino (2003a; 2005) es una de las opciones más sólidas porque incluye todos los elementos que intervienen en la misma: personal administrativo de las instituciones, profesorado y estudiantes. 
doi: http://dx.doi.org/10.15359/ree.23-2.14

URL: http://www.una.ac.cr/educare

CORREO: educare@una.cr

La alfabetización académica propone la enseñanza de la comprensión lectora situada en la disciplina y desarrollada en las asignaturas específicas de cada carrera o programa de estudios a fin de que tenga un sentido integral en la formación del estudiantado, desde un punto de vista lingüístico, discursivo, conceptual y contextual (Caldera y Bermúdez, 2007; Gatti, Grinsztajn, Cética y Vaccaro, 2014). Sin embargo, debemos aceptar que ha sido complejo llevar a cabo los diferentes pasos de implementación de las estrategias que involucran la lectura a través del currículo, por todo lo que conlleva convencer al personal docente de las asignaturas o cursos disciplinares de enseñar a leer y a escribir, desde sus propios contenidos y, además, capacitarlo adecuadamente, entre otras cosas (Carlino, 2013); mientras tanto nuevos grupos de estudiantes siguen arribando a las aulas universitarias y continúan presentando estas dificultades que obstaculizan sus estudios.

Ante esta perspectiva, provisoriamente podría recurrirse a la utilización de las estrategias que ya están aplicándose en las universidades para solucionar los conflictos de lectura y escritura: los cursos de lenguaje, los cursos remediales o de regularización y los centros de escritura, pero añadiendo una perspectiva aún más integral con respecto a la escritura académica, a fin de propiciar ya no únicamente la comprensión general sino configurar las capacidades estudiantiles para una comprensión especializada.

Para ayudar a desarrollar la comprensión especializada se podría partir de una orientación concreta para que, en los textos académicos, el sujeto lector identifique el género discursivo, el objetivo autoral, las tesis e ideas principales planteadas, la intertextualidad; así mismo se podría hacer énfasis en el manejo de vocabulario especializado y en el conocimiento de los conceptos teóricos disciplinares. Por tanto, a continuación se proponen algunos objetivos de aprendizaje que podrían perseguirse en la clase de lenguaje, en los cursos de nivelación o remediales, en los centros de escritura así como en los cursos y asignaturas disciplinares.

a. Reconocer el tipo de género discursivo del texto. Como ya se mencionó, los textos académicos tienen funciones y formas diversas que los distinguen y agrupan; lo cual requiere del público lector identificar la estructura del texto y los tipos de secuencias discursivas utilizadas en él. Esto le permite tener mayor claridad sobre de qué manera será tratado el tema o el objeto de estudio del texto y puede aplicar estrategias definidas de lectura -como predicciones, inferencias y lectura entre líneas- para comprender su sentido global de manera eficaz. El sujeto lector necesita conocer los textos universitarios en su complejidad; debe considerar que la información presentada ha pasado por un proceso de selección y organización y que está redactada con diferentes estrategias lingüísticas y discursivas. La conciencia de estos mecanismos de disposición formal es indispensable para la comprensión especializada. 
b. Examinar los objetivos o propósitos autorales. Debe hacerse notar que quienes escriben los textos académicos, en tanto autoridades de un área o disciplina, escriben con propósitos definidos, aunque no siempre sean explícitos. Los textos universitarios tienen un interés científico, pero su forma y contenido están organizados, según lo que su autoría pretende realizar con la información que presenta. El público lector debe estar comprometido a desentrañar y analizar esos objetivos a fin de obtener una comprensión total y útil del texto.

c. Identificar la tesis o idea central que defiende cada texto. El objetivo del autor o autora conduce al meollo central del texto, a la propuesta de una idea, una tesis o una acción específica. Reconocer cuál es el objeto de la escritura le da sentido al acto mismo de leer y propicia una comprensión global del texto. Se debe promover que la persona que lee comprenda estos temas -tratados con un interés científico y desde una perspectiva disciplinar- haciendo varias operaciones como contextualizar la materia tratada, examinar las propuestas presentadas, abstraer conceptos o teorías e incluso tomar una posición con respecto a un debate. Es decir, encontrar la tesis central de un texto académico requiere una lectura profunda e inferencial, producto del análisis crítico del texto mismo, su contexto y sus paratextos.

d. Identificar las ideas principales que constituyen el entramado discursivo de quien escribe para presentar su idea central o tesis. Se debe mostrar que tanto los objetivos del escritor o escritora como su propuesta están expuestos en una serie de postulados o ideas que quien lee debe, en primera instancia, reconocer dentro de la exposición o argumentación del texto; asimismo, se debe propiciar que sea capaz de reconstruir la organización y jerarquización de estas en la estructura del propio texto, y que valore la función desempeñada por ellas para los propósitos de quien lo escribió. El público lector universitario debe analizar el texto académico desde su composición, a fin de familiarizarse con los modos de expresión y organización de los textos de su disciplina.

e. Diferenciar las relaciones de intertextualidad. Los textos utilizados en la educación superior implican un diálogo constante entre autoridades, postulados y comunidades discursivas; lo cual se refleja en las referencias a otras ideas, teorías o autoridades de la materia que abordan y que se aluden en cada texto. Hay que motivar al sujeto lector a identificar las diferentes voces que participan en ese diálogo, reconociendo sus intervenciones y sus particulares sistemas de citación; también a a registrar el tipo de función que cumplen esas referencias en el texto leído (sustentar, apoyar o debatir) así como determinar el grado de su relación con esos otros textos, a partir de las menciones, paráfrasis o citas directas de estos. No basta solo con reconocer lo que dice el escritor o la escritora leída, es necesario tener conciencia de que en ese mismo texto hay un entramado de textos, los cuales lo enriquecen y ayudan a darle sentido según su propósito y propuesta. Quien lee textos universitarios debe incorporarse a ese diálogo para comprender las profundidades de su tema y disciplina plasmadas en un escrito. 
doi: http://dx.doi.org/10.15359/ree.23-2.14

URL: http://www.una.ac.cr/educare

CORREO: educare@una.cr

f. Reconocer la especificidad de los conceptos, metodología y prácticas de su disciplina concreta. Las disciplinas desarrollan y emplean su propia visión del conocimiento de sus áreas de interés; esto implica que sus textos giran en torno a tradiciones, metodologías y prácticas basadas en conceptos disciplinaresya establecidos o en proceso de establecimiento. El estudiantado que ingresa a la universidad debe integrarse a este universo conceptual lo más pronto posible a fin de dominar los textos concretos en todos sus niveles de comprensión.

g. Conocer el vocabulario o léxicoespecífico de la comunidad.En elámbito universitario, los textos contienen términos lexicales complejos que pueden resultar desconocidos por el estudiantado no iniciado y obstaculizan su comprensión. Cuando se trata de la disciplina específica en la que se está formando, se debe propiciar el conocimiento del significado de las palabras que conforman el discurso, pues eso ayuda a comprender, de manera literal, su contenido, pero también lo ubica dentro del marco disciplinar, temático y discursivo que se requiere para analizar la información y aplicarla a su práctica universitaria, profesional e incluso personal. Manejar el vocabulario particular de su área, le ofrece herramientas para la apropiación del conocimiento.

Con tales objetivos de aprendizaje, los cursos ofrecerían al estudiantado de nuevo ingreso un panorama acerca de la nueva cultura a la que se incorpora, presentando el lenguaje, los discursos, conceptos e ideas específicos que caracterizan esta forma de expresión; al mismo tiempo, enfatizarían el propósito de continuar el proceso de desarrollo de la competencia de lectura (y escritura), a partir de la presentación, utilización y análisis de textos académicos con la guía del profesorado de lenguaje ayudándoles así a superar las dificultades que tales escritos suscitan y preparándolos de manera concreta para los cursos disciplinares. Esto puede incorporarse a la regularización o nivelación en los aspectos más débiles de la comprensión y haciendo énfasis en aquellos que presentan más dificultades, según se presenta en la prueba diagnóstica que ilustra este trabajo.

Estemodelosería complementarioal delaalfabetizaciónacadémica.Sibienesindispensable enseñar a leer y a escribir desde y para las asignaturas concretas de cada disciplina, implementar estos objetivos de aprendizaje desde los cursos de lenguaje o remediales, partiendo de una perspectiva de inmersión en la cultura y textos académicos, podría significar un cambio efectivo en la adaptación de estudiantes al mundo universitario, pues intervendría inmediatamente sobre las dificultades que impiden su comprensión especializada. Esto aportaría un recurso plausible al momento de diseñar cursos orientados a iniciar una inculturación a la universidad teniendo como centro las características y exigencias precisas de los textos académicos. 


\section{Referencias}

Caldera, R. y Bermúdez, A. (2007). Alfabetización académica: Comprensión y producción de textos. Educere, 11(37), 247-255. Recuperado de https://media.utp.edu.co/referenciasbibliograficas/uploads/referencias/articulo/206-alfabetizacin-acadmica-comprensin-yproduccin-de-textospdf-fjGtX-articulo.pdf

Carlino, P. (2003a). Alfabetización académica: Un cambio necesario, algunas alternativas posibles. Educere, 6(20), 409-420. Recuperado de http://www.redalyc.org/articulo.oa?id=35662008

Carlino, P. (2003b). Leer textos científicos y académicos en la educación superior: Obstáculos y bienvenidas a una cultura nueva (1). Uni-Pluri/versidad, 3(2), 17-23. Recuperado de https:// aprendeenlinea.udea.edu.co/revistas/index.php/unip/article/viewFile/12289/11146

Carlino, P. (2005). Escribir, leer y aprender en la universidad. Una introducción a la alfabetización académica. México: Fondo de Cultura Económica.

Carlino, P. (2013). Alfabetización académica diez años después. Revista Mexicana de Investigación Educativa, 18(57), 355-381. Recuperado de http://www.redalyc.org/pdf/140/14025774003.pdf

Carranza, M., Celaya, G., Herrera, J. A. D. y Carezzano, F. J. (2004). Una forma de procesar la información en los textos científicos y su influencia en la compresión. Revista Electrónica de Investigación Educativa, 6(1), 1-15. Recuperado de https://redie.uabc.mx/redie/article/ view/91/157

Cassany, D. y Morales, Ó. A. (2009). Leer y escribir en la universidad: Los géneros científicos. En Cassany, D. (Comp.), Para ser letrados. Voces y miradas sobre la lectura (pp. 109-128). Barcelona: Paidós.

Castelló, M. (2014). Los retos actuales de la alfabetización académica: Estado de la cuestión y últimas investigaciones. Enunciación, 19(2), 346-365. Recuperado de http://revistas. udistrital.edu.co/ojs/index.php/enunc/article/view/8256

Estienne, V. M. y Carlino, P. (2004). Leer en la universidad: Enseñar y aprender una cultura nueva. Uni-pluri/versidad, 4(3), 9-17. Recuperado de https://aprendeenlinea.udea.edu.co/revistas/ index.php/unip/article/view/12210/11075

Fuster, Y. (2016). El texto académico como género discursivo y su enseñanza en la educación terciaria. Palabra Clave, 5(2), 1-11. Artículo e007. Recuperado de http://www.palabraclave. fahce.unlp.edu.ar/article/view/PCv5n2a02 
doi: http://dx.doi.org/10.15359/ree.23-2.14

URL: http://www.una.ac.cr/educare

CORREO: educare@una.cr

Gatti A., Grinsztajn F., Cética, P. y Vaccaro, M. (2014). Alfabetización académica, formación docente y gestión de la enseñanza en la universidad. En XIV Coloquio internacional sobre gestión universitaria. La gestión del conocimiento y los nuevos modelos de universidades (pp. 1-18). Florianópolis: CIGU. Recuperado de https://repositorio.ufsc.br/bitstream/ handle/123456789/132222/2014-450.pdf?sequence=1\&isAllowed=y

Giraldo, G. (2011). Los procesos de lectura y escritura en la inteligencia emocional. Poliantea, 7-13, 205-218. doi: http://dx.doi.org/10.15765/plnt.v7i13.146

González, B. Y. y Vega, V. (2012-2013). Lectura y escritura en la educación superior colombiana: Herencia y deconstrucción. Revista Interacción, 12, 195-201. Recuperado de http://www. unilibre.edu.co/revistainteraccion/

Henao-Salazar, J. I., Londoño-Vásquez, D. A., y Frías-Cano, L. Y. (2014). Leer y escribir en la universidad: El caso de la Institución Universitaria de Envigado. Íkala, Revista de Lenguaje y Cultura, 19(1),27-45. Recuperado de http://www.redalyc.org/articulo.oa?id=255032609003

Kintsch, W. y Van Dijk, T. A. (1978). Toward a model of text comprehension and production. Psychological Review, 85(5), 363-394. doi: https://doi.org/10.1037/0033-295X.85.5.363

Marín, M. (2007). Lectura de textos de estudio, pensamiento narrativo y pensamiento conceptual. Holográmica, 4(7), 61-80. Recuperado de http://www.cienciared.com.ar/ra/usr/3/523/n7 vol4pp61 80.pdf

Martínez, M. C. (1997). El desarrollo de estrategias discursivas a nivel universitario. En M. C. Martínez (Comp.), Los procesos de la lectura y la escritura (pp. 11-41). Santiago de Cali: Universidad del Valle.

Mateos, M. (2009). Aprender a leer textos académicos: Más allá de la lectura reproductiva. En J. I. Pozo y M. Pérez (Coords.), Psicología del aprendizaje universitario. La formación en competencias (pp. 106-119). Madrid: Ediciones Morata.

Pérez, M. y Rincón, G. (Coords.). (2013). ¿Para qué se lee y se escribe en la universidad colombiana? Un aporte a la consolidación de la cultura académica del país. Bogotá: Pontificia Universidad Javeriana. Recuperado de http://wac.colostate.edu/books/colombian/highered.pdf

Riestre, D. (2006). Usos y formas de la lengua escrita. Reenseñar la escritura a los jóvenes. Un puente entre el secundario y la universidad. Buenos Aires: Ediciones Novedades Educativas.

Salazar-Sierra, A., Sevilla-Rengifo, O., González-Pinzón, B., Mendoza-Arciniegas, C. E., EcheverriGuzmán, A., Quecán-Castellanos, D. ... Lozano-Ramírez, M. (2015). Lectura y escritura en la universidad: Contribución para reconstruir una historia. Magis. Revista Internacional de Investigación en Educación, 8(16), 51-70. doi: http://dx.doi.org/10.11144/Javeriana.m8-16.leuc 
Teberosky, A. (2007). El texto académico. En M. Castelló (Coord.), Escribir y comunicarse en contextos científicos y académicos: Conocimientos y estrategias (pp. 17-46). Barcelona: Graó.

Ugarriza, N. (2006). La comprensión lectora inferencial de textos especializados y el rendimiento académico de los estudiantes universitarios del primer ciclo. Persona, 9, 31-75. doi: https:// doi.org/10.26439/persona2006.n009.902

Van Dijk, T. A. (1983). La ciencia del texto. Barcelona: Paidós.

Van Dijk, T. A. (2010). Discurso, conocimiento, poder y política. Hacia un análisis crítico epistémico del discurso. Revista de Investigación Lingüística, 13, 167-215. Recuperado de http:// revistas.um.es/ril/article/view/114181

Van Dijk, T. y Kintsch, W. (1983). Strategies of discourse comprehension. New York: Academic Press.

Velásquez, M., Cornejo, C. y Roco, Á. (2008). Evaluación de la competencia lectora en estudiantes de primer año de carreras del área humanista y carreras del área de la salud en tres universidades del Consejo de Rectores. Estudios Pedagógicos, 34(1), 123-138. doi: https:// doi.org/10.4067/S0718-07052008000100007

Vidal-Moscoso, D. y Manríquez-López, L. (2016). El docente como mediador de la comprensión lectora en universitarios. Revista de la Educación Superior, 45(177), 95-118. Recuperado de https://doi.org/10.1016/j.resu.2016.01.009

Zanotto, M. (2016). Comprensión lectora y aprendizaje de textos académicos: Hacia una lectura estratégica en el campo de las ciencias sociales. En G. Bañales Faz, M. Castelló Badía y N. A. Vega López, (Coords.), Enseñar a leer y escribir en la educación superior. Propuestas educativas basadas en la investigación (pp. 29-52). México: Editorial SM. Recuperado de http://www.fundacion-sm.org.mx/sites/default/files/Ense\%C3\%B1ar\%20a\%20leer\%20 \%20escribir.pdf 